\title{
HOUSING OF PHOTOELECTRIC EQUIPMENT IN ROCKET EXPERIMENTS
}

\author{
H. HESSBER G \\ Max-Planck-Institut für Physik und Astrophysik, Institut fïr extraterrestrische Physik, \\ Garching, Germany
}

The housing of photoelectric equipment in rocket experiments results among others in the following demands: Stability of the structure and nevertheless little weight, exact geometrical alignment and at the same time soft, vibration- and shock-protecting supportings (in case of photomultipliers for instance).

Solution of these self-contradicting features in case of experiment R-130 was as follows: The delicate photomulipliers (PM) were supported in a double damping system. First they were potted with silicon-rubber inside a thin-wall metal tube. This tube then allowed (besides the electrostatic screening and insulating effect) a more rigid and convenient clamping inside the overall structure (Figure 1). Inside this structure the PM were fitted in bridges between the shell-parts.

In these bridges sustaining again was given by casted silicon rubber rings, one at the front and one at the rear end of the PM. With that a system of three elastic elements was given. By independent variation of the hardness of the rubbers, a set could be found, that yielded very good damping of vibrations with a very flat resonance characteristic. And that in spite of the very resonance-susceptible bridge mounting. The remaining displacement of the PM by any environmental influence was much smaller than $1 \mathrm{~mm}$.

These features were worked out with a metal structure. Experiments have been made with a casted overall structure, only the flanges being made of metal. The casting material is fibre reinforced epoxy. Glas fibres are in the shells and polyamid fibres in the bridges. In both cases the specific weight was kept below unity by special filling substances. Thereby it could be shown that the fibre reinforcing not necessarily must lead to higher specific weight of the final product. The manufacturing process only needs conventional laboratory equipment and thus at the same time injection diecasting and its necessary very high-costly machines were avoided. The productionmethod opens the possibility for small quantity manufacturing of fibre reinforced dies at about the same price that would have been to be paid for metal structures anyway. Already the second model with-stood sinoidal vibration levels of $40 \mathrm{~g}$ at the input with all electronic and multiplier-dummies fitted to it.

The two major advantages of the epoxy-parts are little weight and better damping of vibrations. Compared to a magnesium alloy metal structure of the same dimensions (including flanges) the weight could be diminished by $35 \%$; presumably this is not yet the limit. Resonance amplitudes were diminished and shifted to lower frequencies. In general. the epoxy structure behaves softer towards vibrations and thereby reduces the stress on delicate electronics and optical elements. 


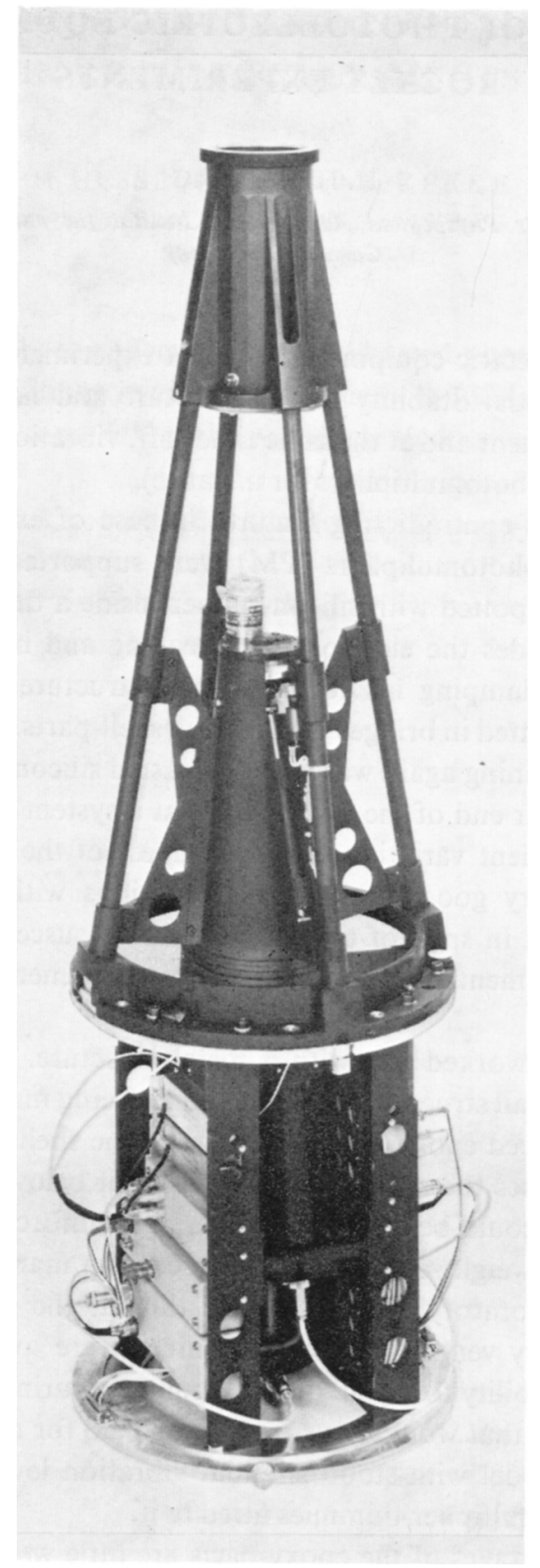

Fig. 1. Prototype Experiment R-130 (VUV-Starphotometer). Upper part: Telescope: lower part: Metal structure with photomultipliers (in between) and electronics (sides). Overall size about $1 \mathrm{~m}$. 


\section{DISCUSSION}

B. W. Campbell: (1) You mentioned that there were errors in the absolute calibration due to switching the high voltage supply on above the atmosphere. Do you know the percentage error and how does it vary with type of photocathode.

(2) Are the photomultipliers operated with the cathode above earth potential.

(3) Who makes the corona-proof connectors and what is the ultimate vacuum at which they can be operated. Why was it necessary to further encapsulate the EMR detectors.

$H$. Hessberg: (1) The error has two major sources, dark current of the PM and run-in of the highvoltage source. The dark current is dangerous for low level signals and depends upon PM prehistory before switch-on and upon photocathode-type, in our case errors as high as $100 \%$ could result. The run-in of high-voltage source would result in errors up to $10 \%$.

(2) No, the PMs are 'tilted', that is the cathode is on 'earth'-potential.

(3) The corona-tight connectors are manufactured by Fa. Dr Spinner GmbH, 8 München, Erzgiessereistr. 33, Germany. The ultimate test-vacuum was $10^{-5}$ Torr for several hours, the limit was given by the application and is not believed to be the ultimate one.

(4) We encapsuled the EMR-multipliers in metal housings for mechanical damping and electrical screening reasons. 